\title{
Neuroprotection of multifunctional phytochemicals as novel therapeutic strategy for neurodegenerative disorders: antiapoptotic and antiamyloidogenic activities by modulation of cellular signal pathways
}

\author{
Makoto Naoi*,1, Masayo Shamoto-Nagai ${ }^{1}$ \& Wakako Maruyama ${ }^{1}$ \\ ${ }^{1}$ Department of Health \& Nutrition, Faculty of Psychological \& Physical Science, Aichi Gakuin University, Nisshin, Aichi, Japan \\ *Author for correspondence: Tel.: +81 056173 1111, Ext. 3494; Fax: +81 56173 1142; mnaoi@dpc.agu.ac.jp
}

In neurodegenerative disorders, including Alzheimer's and Parkinson's disease, neuroprotection by diet and natural bioactive compounds has been proposed to prevent the onset and progress of neurodegeneration by modification of pathogenic factors. Plant food-derived phytochemicals protect neurons via targeting oxidative stress, mitochondrial dysfunction, neurotrophic factor deficit, apoptosis and abnormal protein accumulation. This review presents the molecular mechanism of neuroprotection by phytochemicals: direct regulation of mitochondrial apoptotic machinery, modification of cellular signal pathways, induction of antiapoptotic $\mathrm{Bcl}-2$ protein family and prosurvival neurotrophic factors, such as brain- and glial cell line-derived neurotrophic factor, and prevention of protein aggregation. Multitargeted neuroprotective agents are under development based on the structure of blood-brain barrier-permeable phytochemicals to ameliorate brain dysfunction and prevent neurodegeneration.

First draft submitted: 30 August 2018; Accepted for publication: 26 November 2018; Published online: 19 February 2019

Keywords: Alzheimer's disease $\bullet$ antioxidant $\bullet$ apoptosis $\bullet$ autophagy $\bullet$ cellular signaling • mitochondria • neuroprotection • neurotrophic factor • Parkinson's disease • phytochemicals • protein aggregation

In our aging society, increase of patients with age-associated neurodegenerative disorders is an oppressive issue for medical care, society and economy. The etiology of two most common neurodegenerative disorders, Alzheimer's disease (AD) and Parkinson's disease (PD), has not been clarified. However, the common pathological hallmarks have been associated with these diseases: loss of distinct population of neurons and accumulation of disease-specific inclusion bodies. New therapeutic strategy is now proposed to target multiple pathogenic factors, including oxidative stress, mitochondrial dysfunction, impaired homeostasis of energy, transition metals (iron, copper), calcium and hormones (insulin, estrogen for women), deficits of neurotrophic factors (NTFs), inflammation, accumulation of modified proteins and activation of programmed cell death. These factors are regulated by interaction between the genetic and environmental factors, and the molecular mechanism has been intensively investigated.

Neuroprotection is aimed to prevent neuronal death, regenerate neuronal network and ameliorate the brain dysfunction as 'disease-modifying' therapy in $\mathrm{AD}, \mathrm{PD}$ and depression. Quite a huge number of compounds, including inhibitors of monoamine oxidase (MAO) (selegiline rasagiline), antioxidants (vitamin E, C, transient metal chelators), bioenergetic compounds (coenzyme Q) and anti-inflammatory agents, have been reported to be neuroprotective, but clinical studies have not been able to prove fully the prevention of disease progression [1].

Epidemiological and clinical intervention studies have presented that diet habits, such as Mediterranean diet, show beneficial effects in patients with dementia, $\mathrm{AD}$, mild cognitive impairment, $\mathrm{PD}$ and depressive disorders [2,3]. Components of Mediterranean diet, phytochemicals (the secondary metabolites of plants) and $\omega-3$ polyunsaturated 
fatty acids, were reported to have maintained cognitive function in human studies. The total low level of urinary polyphenols has been correlated with the risk of cognitive decline in older adults [4].

Herbal preparation and phytochemicals isolated from plant food have been proposed as 'herbal medicine' for the treatment of PD [5] and AD [6]. Flavonoids found in high quantities in vegetables, fruits, tea, red wine and chocolate, and resveratrol enriched in red wine were reported to improve cognitive function in clinical trials, which, however, has been not fully established $[7,8]$. Flavonoids are strong antioxidants via direct scavenge of reactive oxygen and nitrogen species (ROS, RNS), upregulation of ROS-removing enzymes and downregulation of radical-producing ones. However, the neuroprotective activity does not directly depend on the antioxidant activity, and new molecular mechanisms are now proposed for promotion of neuronal survival.

This review presents the recent results on neuroprotective intervention by phytochemicals to the pathogenic factors of neurodegeneration: oxidative stress, neuroinflammation, mitochondrial dysfunction, deficit NTFs and accumulation of abnormal-modified protein. Phytochemicals can directly inhibit or promote mitochondrial apoptosis cascade, which might be associated with the ambivalent functions in decision of cell survival [9]. Phytochemicals modulate cellular signal pathways linking to the expression of antiapoptotic Bcl-2 and NTFs, such as brain-derived and glial cell line-derived NTF (BDNF, GDNF), and show NTF-like activities in the brain [10,11]. Phytochemicals activate the major proteolysis pathways in neuron, the ubiquitin-proteasome system (UPS) and autophagy-lysosome pathway (ALP), to prevent accumulation of disease-specific abnormal protein aggregates and production of toxic oligomer and fibril [12]. These multiple neuroprotective activities of phytochemicals are discussed in relation to the development of new therapeutic strategy for neurodegenerative disorders. Synthesis of novel multitargeted neuroprotective compounds based on the structure of phytochemicals is also discussed in order to develop novel therapeutic agents capable of inhibiting MAO and acetylcholinesterase (AChE), preventing protein aggregation and inducing NTF expression.

\section{Phytochemicals: the classification, structure \& antioxidative \& anti-inflammatory functions}

Polyphenols are present in plants, fruits and vegetables, and flavonoids are the largest groups of $>6000$ established species. Figure 1 shows the classification and chemical structure of major flavonoids and other phytochemicals discussed in this review. Oxidative stress is caused by excess production of ROS-RNS by mitochondria and NADPH oxidase, and decline in antioxidant activity in cells, and it is an early and critical event in PD, AD, amyotrophic lateral sclerosis and multiple sclerosis [13,14]. Free radicals oxidize protein, RNA and DNA, peroxide membrane lipids, damage membrane and induce proteins crosslinking. The antioxidant activity of catechol structure-containing flavonoids depends on the number of hydroxyl groups in the aromatic A and B rings, and the presence of 2,3unsaturation and a 4-carbonyl in the $\mathrm{C}$ ring. Flavonoids donate a hydrogen molecule, form a phenoxyl radical and scavenge single oxygen, superoxide, hydroxyl and peroxyl radicals by release of another hydrogen. The diol group forms a complex with ferric iron, copper and other transition metal ions, and prevents ROS production [15]. A marine carotenoid astaxanthin scavenges singlet oxygen, hydroxyl and peroxy radicals and nitric oxide. It spans through the membrane lipid bilayers, traps radicals at the conjugated polyene chain and in the terminal ring moiety, and removes lipid peroxide products from the membrane [16]. The antioxidant potency of phytochemicals, especially flavonoids, has been proposed to contribute to neuroprotection primarily in vitro and in vivo. However, the in vivo antioxidant actions of polyphenols require the concentrations of 10-100 $\mu \mathrm{M}$ range, one or two order higher than those in the plasma. The structure required for neuroprotection is not always correlated with that for antioxidant function. The ortho-dihydroxy substitution in B ring of flavones is not necessary for neuroprotection, whereas the hydroxyl substitution in the position 3 on the $\mathrm{C}$ ring and in position 5 on the $\mathrm{A}$ ring is required for neuroprotective activity of quercetin [17]. These results suggest the involvement of antioxidant-independent mechanism in neuroprotection.

Polyphenols upregulate ROS-scavenging enzymes, like catalase, superoxide dismutase, glutathione peroxidase, glutathione $S$-transferases, glutathione reductase and $\mathrm{NAD}(\mathrm{P}) \mathrm{H}$ : quinone oxidoreductase (NQO1). The induction is mediated by activation of cellular signal pathways as discussed below. Flavonoids (apigenin, luteolin, kaempferol) inhibit ROS-producing xanthine oxidase, and polyphenols from blue berry and amentoflavone suppress the expression of inducible nitric oxide synthase. Flavonoids (baicalein, quercetin, myricetin, EGCG) and nonflavonoids (gallic, protocatechuic acids) were reported to chelate iron and copper ions, and reduce free radical production.

Chronic inflammation is one of the decisive pathogenic factors in $\mathrm{AD}$ and PD [18]. ROS plays a key role in inflammation by release of inflammatory signal molecules peroxiredoxin 2 (PRDX2) and activates macrophages to release IL-6. Flavonoids, curcumin, lycopene (a linear unsaturated hydrocarbon carotinoid present in tomatoes) 


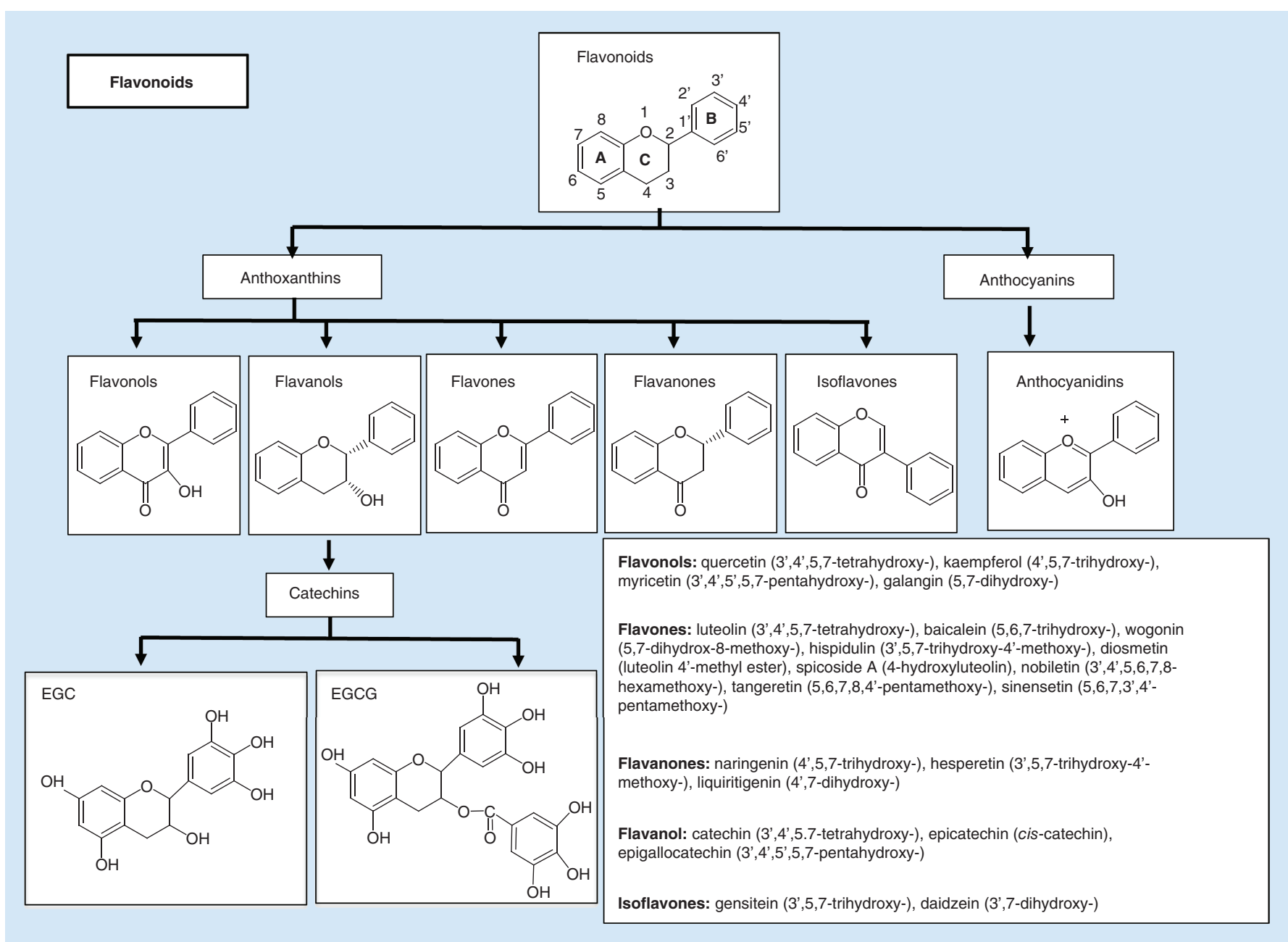

Figure 1. Classification and structure of main classes of phytochemicals. Polyphenols consisting of multiple hydroxyl groups on aromatic rings are categorized into flavonoids, phenolic acids (gallic and ferulic acid), stilbenes (resveratrol), curcumin, astaxanthin, diferoxymethane and tannins. The first four classes are most intensively studied. The structures of epigallocatechin, epigallocatechin gallate and non-flavonoids (astaxanthin, curcumin and sesame lignans) cited in this review are also shown.

and stilbenoids inhibited proinflammatory transcription factors (NF- $\mathrm{KB}, \mathrm{AP}-1$ to suppress synthesis and release of inflammatory TNF- $\alpha$, IL-6, inducible nitric oxide synthase and MCP-1 [19,20]. In in vivo models, resveratrol, curcumin and flavonoids inhibited the activity of arachidonic acid-metabolizing enzymes, COX and lipoxygenase, and suppressed the production of inflammation mediators, arachidonic acid, prostaglandins and leukotrienes, through modulation of the second messengers (cAMP, cGMP, protein kinases and calcium).

\section{Phytochemicals protect mitochondrial function \& increase biogenesis}

Mitochondria are the main energy producers in cells and essential for cellular functions. The defects cause oxidative stress, activate death signal pathways and induce neurodegeneration in aging, AD, PD, Huntington's disease, stroke and psychiatric disorders [21]. In $\mathrm{AD}$, the pathogenic amyloid- $\beta(\mathrm{A} \beta)$ and tau, and in PD $\alpha$-synuclein ( $\alpha$ Syn) are accumulated in mitochondria, and $\mathrm{A} \beta$ inhibits complex IV in the electron transport chain (ETC) and $\alpha$ Syn deregulates complex I. In PD, the deficiency of complex I in the ETC was reported [22], and a dopaminergic neurotoxin 1-methyl-4-phenyl-1,2,3,6-tetrahydropyridine (MPTP) inhibited the complex I and induced parkinsonism in humans.

Phytochemicals regulate mitochondrial functions, apoptosis signaling, the ETC activity, ATP synthesis, ROS/RNS production, mitochondrial biogenesis and degradation by autophagy (mitophagy), and exhibit neuroprotection [23]. EGCG, quercetin, myricitrin, luteolin, hesperidin, huperzine (daidzen-8-C-glucoside isolated 

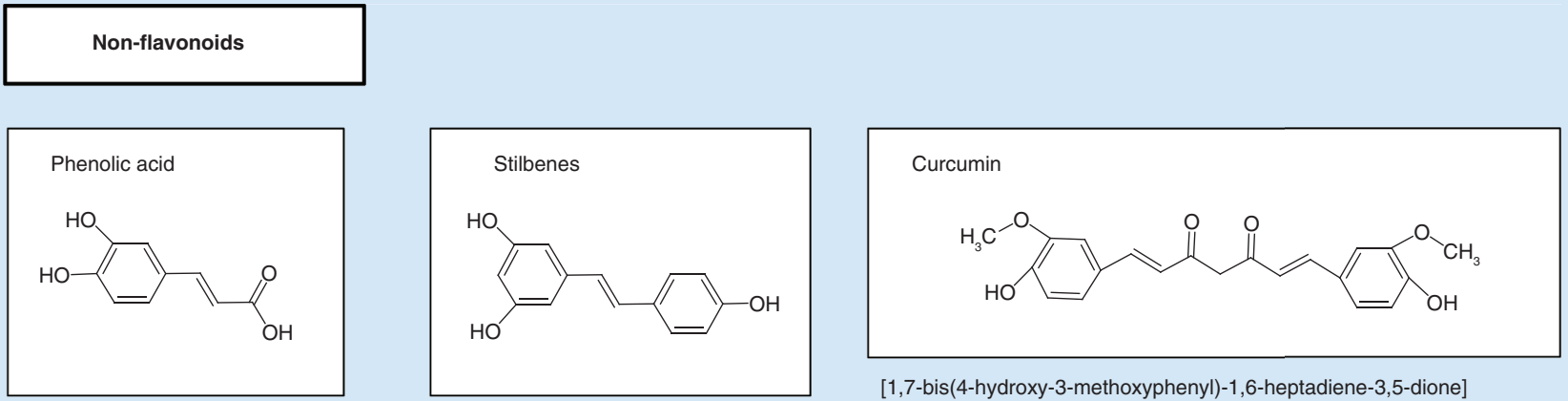

[1,7-bis(4-hydroxy-3-methoxyphenyl)-1,6-heptadiene-3,5-dione]

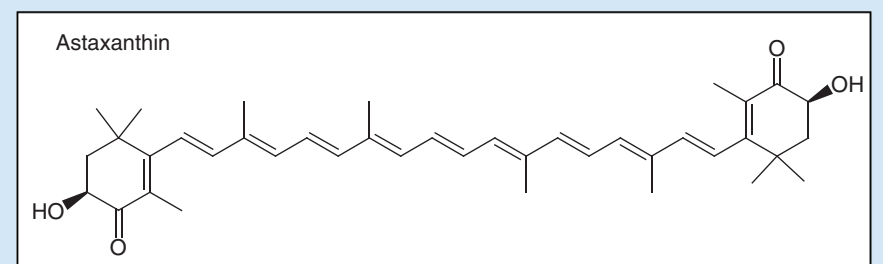

Phenolic acids:

Benzoic acids; gallic (3,4,5-trihydroxy-), protocatechuic (3,4-dihydrox-), altenusin [(5,6-dihydroxy-2-methoxy-phenyl)-hydroxy-4methoxy-)

Cynnamic acids; cafferic (3,4-dihydroxy-), ferulic (4-hydroxy-3-methpxy-), p-coumaric (3,4,5-trohydroxy-)

(3,3'-dihydroxy-b-carotnen-4,4'-dione)

Stilbenes: resveratrol (3,5,4'-trihydroxy-trans-)

Sesame lignans
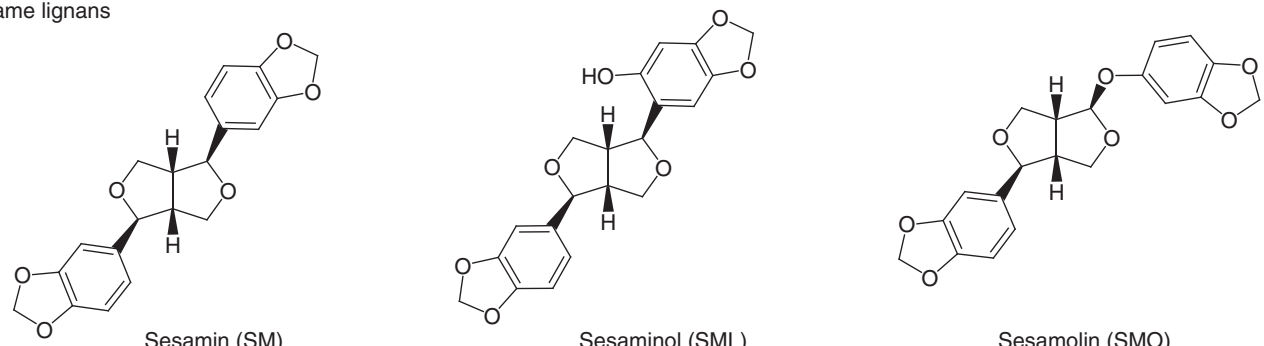

Sesamolin (SMO)

Figure 1. Classification and structure of main classes of phytochemicals (cont.). Polyphenols consisting of multiple hydroxyl groups on aromatic rings are categorized into flavonoids, phenolic acids (gallic and ferulic acid), stilbenes (resveratrol), curcumin, astaxanthin, diferoxymethane and tannins. The first four classes are most intensively studied. The structures of epigallocatechin, epigallocatechin gallate and non-flavonoids (astaxanthin, curcumin and sesame lignans) cited in this review are also shown.

from Huperzia serrata), hesperetin-rutinoside (a flavanone glycoside found in citrus fruits) and proanthocyanidins improved the ETC activity [24].

Mitochondria are dynamic organelles and the quality control is carried out through sequencing damaged organelle by fission (division) and exchanging matrix protein by fusion. Anthocyanins and resveratrol protected complex I and modulated mitochondrial fission/fusion pathways in SH-SY5Y cells transfected with amyloid precursor protein (APP) Swedish K670N/M671L double mutation (APPswe) [25]. Liquiritigenin induced mitochondrial fusion and inhibited mitochondrial fragmentation and cytotoxicity induced by A $\beta$ in SK-N-MC cells [26]. Resveratrol improved the expression of fusion-mediating optic atrophy protein (Opa1), mitofisin-1 and -2 (Mfn1, Mfn2), and fissionregulating dynamin-related protein 1 (Drp1) and mitochondrial fission protein 1 (Fis1), and protected PC12 cells against rotenone-induced cytotoxicity [27].

Phytochemicals increase mitochondrial biogenesis to protect neurons [28]. Mitochondrial biogenesis is regulated by the proliferator-activated receptor PGC- $1 \alpha$ and NRF-1 and -2 , whereas mitochondrial transcription factor A (TFAM) regulates mtDNA replication and transcription. PGC- $1 \alpha$ activates NRF- 1 and -2 transcription factors and estrogen-related receptor $\alpha$ and induces expression of nuclear DNA-encoded mitochondrial protein. PGC$1 \alpha$ activity is post-transcriptionally regulated by phosphorylation and acetylation. Sirt-1 (a nicotinamide adenine dinucleotide (NAD)-dependent deacetylase) deacetylates PGC-1 $\alpha$ and regulates the activity. EGCG and resveratrol were reported to upregulate Sirt-1 and 5'-AMP-activated protein kinase (AMPK), enhance mitochondrial biogenesis via PGC-1 $\alpha$, and protect neuronal cells [29]. Quercetin, hydroxytryrosol (3,4-dihydroxy-phenylethanol present in 


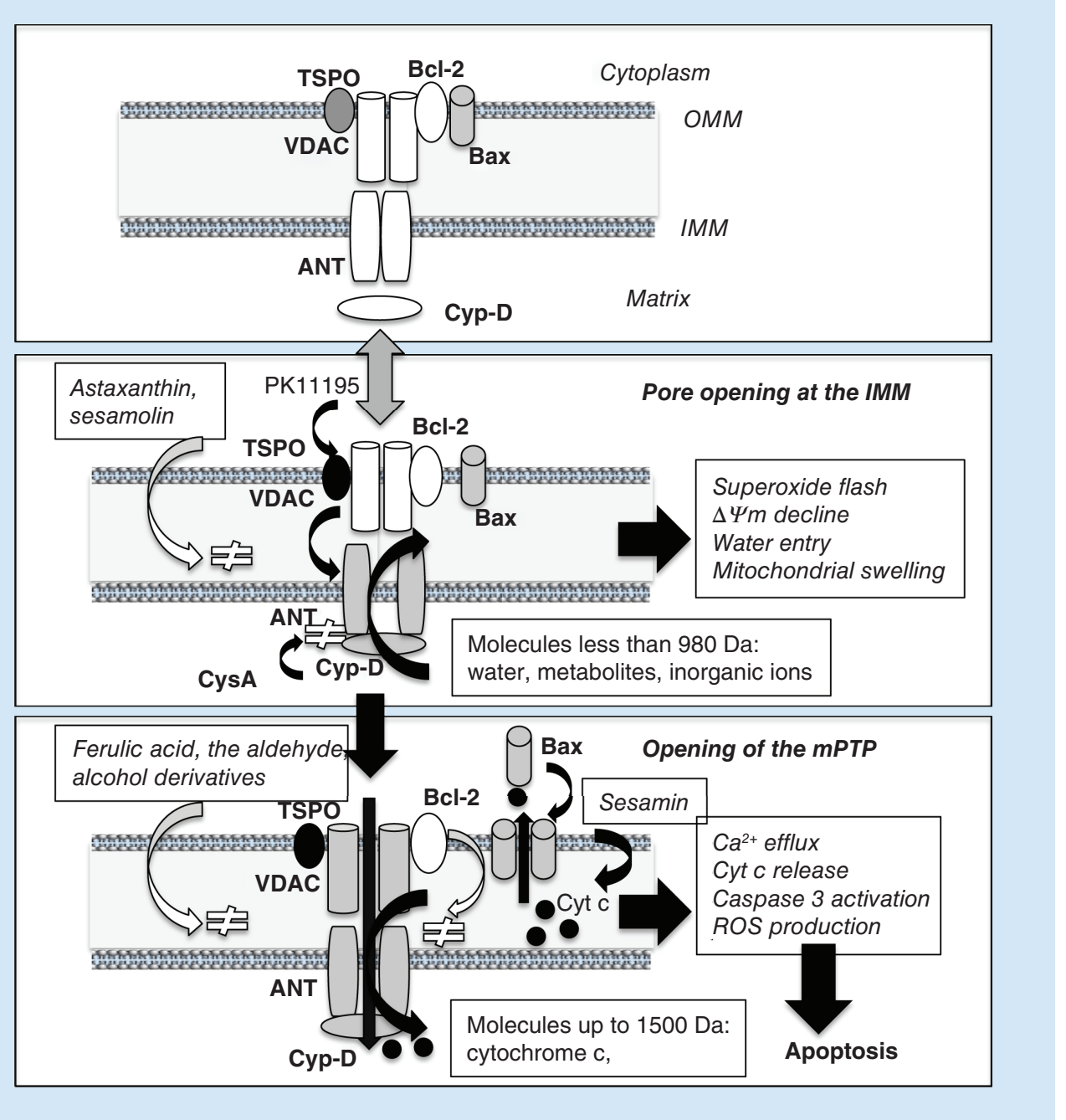

Figure 2. Schematic process of mitochondrial permeabilization to activate apoptosis signaling pathways, and the effects of phytochemicals on the mitochondrial permeability transition pore formation. PK11195 opens the transitional, reversible pore at the inner mitochondrial membrane, then the irreversible pore, mPTP and activates apoptosis cascade. Astaxanthin and sesamolin prevent the pore formation at the inner mitochondrial membrane, and ferulic acid and its aldehyde and alcohol derivatives inhibit the MPTP formation. Sesamin and ferulic acid ethyl ester enhance the mPTP opening. Bcl-2 forms a heterodimer with Bax and suppresses the cytotoxicity. In apoptosis, Bax is dissociated from the heterodimer with Blc-2 and forms the homodimer and pore called 'mitochondrial apoptosis-induced channel' at the outer mitochondrial membrane. At present, direct effect of polyphenols on mitochondrial apoptosis-induced channel formation has not been reported.

IMM: Inner mitochondrial membrane; OMM: Outer mitochondrial membrane; mPTP: Mitochondrial permeability transition pore.

olives), daidzein, genistein, baicalein and wogonin also induced mitochondrial biogenesis. Quercetin was reported to increase the number of muscle mtDNA and promote exercise performance in adult men [30].

\section{Phytochemicals intervene mitochondrial cell death cascades}

In neurodegenerative disorders, apoptosis is a commonly observed type of cell death characterized by the morphological and biochemical changes: cell shrinkage, chromatin condensation and nucleosomal degradation. Apoptosis signal pathways in mitochondria are well conserved and one of the most promising targets of neuroprotection, as presented in Figure 2 and Box 1. Resveratrol, quercetin, rosmarinic acid (a dimer of caffeic acid), astaxanthin and black tea extract prevented membrane permeabilization and protected cells from apoptosis induced by MPTP, amyloid aggregates and ischemia [31]. Recently, we reported that phytochemicals directly prevented or promoted 
Box 1. Mitochondrial apoptosis pathway.

Cell death is classified into apoptosis, autophagy and regulated necrosis. Apoptosis is the most prominent, and its well-conserved process is triggered intrinsically by mitochondrial machinery or extrinsically by receptor-mediated system. Mitochondria-initiated intrinsic apoptosis is activated by death stimuli, like toxins, hypoxia, oxidative stress and deficit of neurotrophic factors.

Mitochondrial apoptotic signal pathway is sequentially activated: increased membrane permeability, formation of the MPTP, efflux of cytochrome $c$ into the cytoplasm from the matrix, assembly of the apoptosome (Apaf-1, caspase-9, ATP), activation of caspase-3, cleaves poly(ADP-ribose)polymerase, nuclear translocation of apoptosis-inducing factor and DNA fragmentation, resulting in apoptosis.

The mPTP opens by step-wise pore formation at the IMM and OMM. Under physiological conditions, mitochondrial membrane permeability is tightly regulated, and mitochondria reserve an electrochemical gradient, membrane potential $(\Delta \Psi \mathrm{m})$, across the IMM. Mild stimulus induces transitional and reversible formation of pore composed of ANT and cyclophilin-D at the IMM. The pore allows the entry of water and solutes with molecular mass up to $980 \mathrm{Da}$ into the matrix, and causes collapse of $\Delta \Psi \mathrm{m}$, cristae reorganization and matrix swelling, with the burst of superoxide production called superoxide flash. Prolonged, more intense insults further open irreversibly the nonselective MPTP at the contact site between the OMM and IMM. The MPTP increases membrane permeability to solutes with molecular mass $<1500 \mathrm{Da}$ and causes the expansion of the matrix and rupture of the OMM.

Structure of the mPTP has not been fully established, but it is composed mainly of VDAC, ANT, TSPO, hexokinases and $\mathrm{BCl}-2$ protein family. The opening is the 'point of no return' in apoptosis.

Cyclosporine A prevents the pore formation of ANT with cyclophilin-D at the IMM. Bcl-2 and Bcl-xL interact with the voltage-dependent anion channel or apoptogenic Bax and Bad, and prevents the MPTP opening at the OMM.

ANT: Adenine nucleotide translocator; IMM: Inner mitochondrial membrane; mPTP: Mitochondrial permeability transition pore; OMM: Outer mitochondrial membrane; TSPO; the outer membrane translocator; VDAC: voltage-dependent anion channel.

the pore formation at mitochondrial membrane in a cellular model of apoptosis induced by PK11195, a ligand of the outer membrane translocator (TSPO) [9]. Astaxanthin, lipophilic derivatives of ferulic acid and sesamolin inhibited formation of pore composed of adenine nucleotide translocator (ANT) and cyclophilin-D at the inner mitochondrial membrane, which was detected as the burst of superoxide production called 'superoxide flash'. The aldehyde and alcohol derivative of ferulic acid suppressed the pore opening at the outer mitochondrial membrane and prevented formation of the mitochondrial permeability transition pore (mPTP), efflux of apoptogenic cytochrome c (Cyt c) and calcium, and apoptosis. On the other hand, hydrophilic ethyl ester of ferulic acid and sesamin promoted the mPTP formation and apoptosis. Phytochemicals, especially curcumin, directly interacted with voltage-dependent anion channel, prevented oxidative modification of the critical thiol residues in ANT and membrane protein, and phosphorylation of voltage-dependent anion channel by glycogen synthase kinase 3 (GSK3), and prevented the pore opening. Flavonoids (kaempferol, bailarein, quercetin, naringenin, EGCG) activated signal pathways to induce antiapoptotic Bcl-2 and Bcl-xL and suppress apoptogenic Bax and Bak, and modulated apoptosis pathway in mitochondria.

On the other hand, quercetin, luteolin, kaempferol, EGCG, theaflavins, baicalein, wogonin, resveratrol and curcumin opened the mPTP and induced apoptosis in cancer cells, through inhibition of Bcl-2 and Bcl-xL, induction of Bax oligomerization, and downregulation of NF- $\mathrm{B}$ signal pathway [32]. Genistein oxidized the thiol groups and pyridine nucleotides and induced mitochondrial permeability transition [33]. The ambivalent functions of polyphenols in mitochondrial death signal pathways have been considered to depend on their concentrations, antioxidant or prooxidant activities, redox state and amphipathic properties [34].

\section{Phytochemicals present NTF-like activity by binding to NTF receptors \& activating signal pathways for neuroprotection}

NTFs play a major role in the development, function and survival of neurons, and the deficiency and gene variation of BDNF and GDNF are associated with pathogenesis of AD, PD and psychiatric disorder (Box 2) [35]. BDNF and GDNF have been proposed as therapeutic targets in neurodegeneration. The administration of GDNF, a dopamine neuron-specific NTF, into the nigra-striatal system has been reported in patients with PD, and the beneficial results were reported in some trials [36]. However, these NTFs cannot penetrate the blood-brain barrier (BBB) and the delivery system has been required for transport of NTFs into the brain. As an alternative practical method, the activation of de novo biosynthesis of BDNF and GDNF by phytochemicals permeable across the BBB has been proposed. Flavonoids (EGCG, puerarin, hesperitin, quercetin, genistein, kaempferol, naringenin), curcumin and 
Box 2. Neurotrophic factors in neuroprotection.

NTFs regulate the development, maintenance, function and plasticity of neurons, and protect neuronal cells from cell death. NTFs are classified into the neurotrophin family (BDNF, NGF, neurotrophin-3 and -4 [NT-3, NT-4]), GDNF family of ligands (GDNF, neurturin, artemin and persephin), neurotrophic cytokines (neurokines) and new numbers, such as cerebral dopamine neurotrophic factor, mesencephalic astrocyte-derived neurotrophic factor, ciliary neurotrophic factor and bFGF.

Neurotrophin binds to two classes of receptor, Trk receptors, a member of tyrosine kinase receptor family, and p75NTR of the TNF receptor family. Activation of Trk receptors promotes neuronal survival, whereas that of p75NTR causes cell death. NGF binds to TrkA, BDNF and NT-4/5 to TrkB, and NT-3 to TrkC receptor, whereas all the NTFs bind to p75NTR with the similar affinity. TrkA receptor is expressed exclusively in cholinergic neurons of the basal forebrain, and TrkB and TrkC receptor in the hippocampus.

Neurotrophin binds to Trk receptor and activates signaling cascades; MAPK, PI3K/Akt and phospholipase C- $\gamma$. MAPK and PI3K/Akt pathway are involved in neurite outgrowth and neuronal survival, whereas phospholipase C- $\gamma$ pathway regulates the intracellular $\mathrm{Ca}^{2+}$ levels. BDNF occurs ubiquitously in the brain and particularly markedly in the hippocampus, and is associated with learning, memory and emotion. BDNF protects neuronal populations in $A D, P D$, amyotrophic lateral sclerosis, depression and peripheral sensory neuropathies. Brain BDNF levels decrease in depression.

GDNF is expressed in the striatum (caudate putamen) and thalamus, where catecholaminergic afferent innervation is received. GDNF ligands bind to specific GDNF family receptor $\alpha$ proteins sharing the receptor tyrosine kinase RET. RET activates MAPK, PI3K and phospholipase $C-\gamma$ pathway, and regulates cell survival, differentiation, proliferation, migration, neurite outgrowth and synaptic plasticity. GDNF protects dopaminergic and noradrenergic neurons in vivo and in vitro, and is depleted in the nigral neurons of the parkinsonian brain. GDNF is proposed as a therapeutic agent for PD, amyotrophic lateral sclerosis, drug addiction and alcoholism. Clinical trials to deliver GDNF in the brain have been reported in patients with PD.

AD: Alzheimer's disease; BDNF: Brain-derived neurotrophic factor; bFGF; basic fibroblast growth factor; GDNF: Glial cell line-derived neurotrophic factor; NTF: Neurotrophic factor; PD: Parkinson's disease; RET: rearranged during transfection; Trk: Tropomycin-related kinase.

resveratrol can cross the BBB in agreement with the high lipophilicity, and present NTF-like function in the brain [37]. As summarized in Figure 3, phytochemicals show NTF-mimic activity by binding to NTF receptors, direct activation of downstream signal pathways and induction of NTF expression.

7,8-Dihydroxyflavone (7,8-DHF), 7,8,3'-trihydroxyflavone (7,8,3'-THF), fisetin, deoxygedunin (a derivative of gedunin isolated from Indian neem tree), diosmetin and curcumin were found to bind to BDNF-targeted tropomycin-related kinase B (TrkB) receptor and activate PI3K-Akt (protein kinase B), Ras-MEK (MAPK)-ERK pathways, and finally cAMP-response element-binding protein (CREB) in nuclei. Activation of these pathways has promoted survival of cultured motor neurons, cerebral cortical and hippocampal neuronal cells and spinal ganglion neurons, and also provided antidepressant effects [38]. Nonflavonoid polyphenols activated NGF-specific TrkA receptor. Hesperetin, huperzine, 4,6-dimethoxyphenyanthrene, spicoside A and quinic acid derivatives bound to TrkA receptor, increased neurite outgrowth and showed neuroprotective potency [39]. On the other hand, epicatechin, a selective inhibitor of tyrosine nitration, was reported to inhibit the expression of p75 neurotrophin receptor (p75NTR) and prevent retinal neurodegeneration in a rat model of diabetes [40]. GDNF binds to GDNF family receptor $\alpha$, phosphorylates the receptor tyrosine kinase RET (rearranged during transfection) and activates PI3K and ERK-MAPK pathways, but the binding of polyphenols directly to this receptor has not been reported.

In vitro studies have suggested that polyphenols interact with other receptors, protein or enzymes, and activate signal pathways for neuroprotection. A specific binding site for polyphenols and resveratrol was isolated from plasma membrane in the rat brain [41]. EGCG was found to bind to cell surface-associated $67-\mathrm{kDa}$ laminin receptors, and potentiate neuroprotective and neurogenic action [42]. However, the role of these phytochemical-specific binding proteins in neuroprotection has been only scarcely reported.

MAO is localized at the outer membrane of mitochondria, regulates the homeostasis of monoamine neurotransmitters in the brain, and also serves as the modulator of signal pathways linking to decision of neuronal survival and death [43]. Inhibitors of type A MAO (MAO-A) are applied as antidepressant and anxiolytic agents, whereas type $\mathrm{B}$ (MAO-B) inhibitors (selegiline, rasagiline) are associated with activation of neuroprotective signaling pathways. Flavonoids (catechin, quercetin, naringenin, EGb 761, ginkgolide) inhibited MAO, depending on the presence of a (para-OH-substituted) phenyl at position 2 and unsaturation at the positions 2-3 on the $\mathrm{C}$ ring [44]. Flavonoids with catechol structure (quercetin, ginkgolide B, EGCG) inhibited MAO-A and induced BDNF expression in 


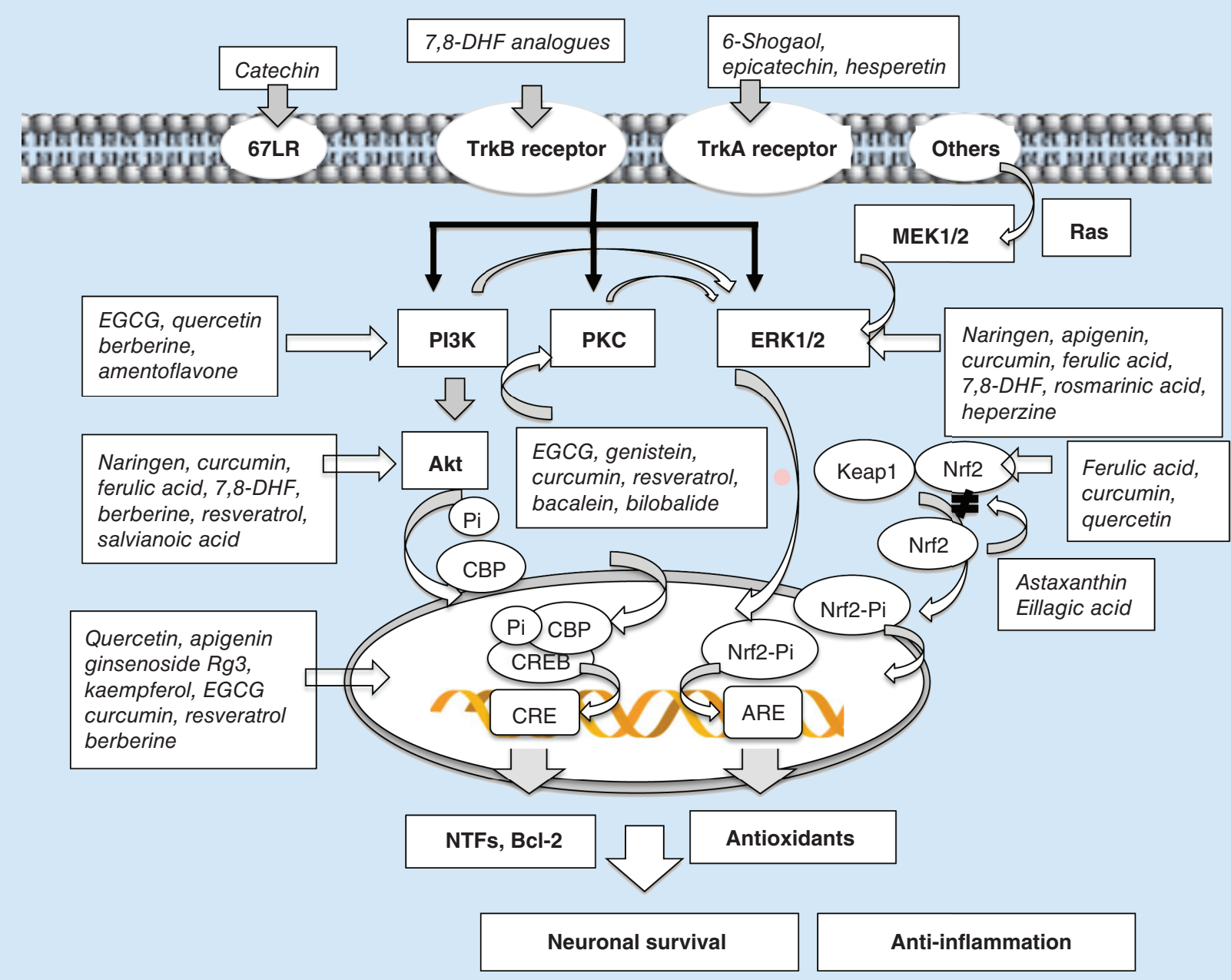

Figure 3. Intracellular targets of phytochemicals to activate the signal transduction and induce neuroprotective gene expression. Phytochemicals bind to receptors of neurotrophic factors receptors (tropomycin-related kinase; TrkB, TrkA) and other binding proteins, activate downstream kinases (PI3K/Akt, PKC, ERK1/2) and phosphorylate CREB protein. Phosphorylated CREB (Pi-CREB) binds to CRE and increases transcription of target genes. Phytochemicals activate Ras-MEK-ERK and Keap-Nrf2-antioxidant response element pathways to increase the expression of antioxidant enzymes.

CREB: CAMP-response element-binding protein.

neuroblastoma SH-SY5Y cells, whereas resveratrol and curcumin inhibited MAO-B functioning as a transcription repressor and increased GDNF in glioblastoma U118MG cells [12].

GABA receptors mediated neuroprotection by anthocyanins against ethanol toxicity in prenatal rat via Bcl2 induction by activation of calcium/calmodulin-dependent protein kinase II (CaMKII)-CREB pathway [45], and that by baicalin (baicalein 7-O-glucuronide) against global ischemia/reperfusion injury in gerbil neurons [46]. Nicotinic acetylcholine receptor $\alpha 4$ and $\alpha 7$ subunits were associated with neuroprotection by EGCG against $A \beta_{1-42-}$ induced cytotoxicity in cultured cortical neurons [47]. Curcumin induced serotonin-1A $\left(5-\mathrm{HT}_{1 \mathrm{~A}}\right)$ receptor [48] and hesperidin $\mathrm{\kappa}$-opioid receptor [49], and they increased BDNF expression and hippocampal neurogenesis and showed antidepressant-like effects. Flavonoids, hesperetin and resveratrol bound to ER $\beta$ and IGF-1 receptor, enhanced BDNF and GDNF expression and promoted cell survival in astrocytes [50].

\section{Phytochemicals modulate cellular signal pathways for neuroprotection}

Phytochemicals activate prosurvival signal pathways through a number of prosurvival MAPK pathways, including PI3K/Akt and PKC (Figure 3). These pathways affect cellular function, synaptic plasticity and memory formation by altering the phosphorylation state of target molecules and modulation of gene expression. Oxidative stress is a 
major stimulus for MAPK cascades, which activate prosurvival ERK and also apoptosis-inducing Jun N-terminal kinase and p38 MAPKs. ERK activation inhibits the death-inducing signaling complex formation and promotes cell survival via transcriptional upregulation of antiapoptotic $\mathrm{Bcl}-2$ and $\mathrm{Bcl}-\mathrm{xL}$, and suppression of Fas-mediated apoptosis.

Hesperetin, naringenin, quercetin, luteolin, amentoflavone, resveratrol and bilobalide derived from Ginkgo biloba leaf have been reported to bind to the ATP-binding site on enzymes and receptors, activate PI3K/Akt and PKC, protect neurons from cell death and ameliorate cognition and memory deficits. Flavonoids activated upstream MAPK-kinase-kinase, prevented Jun $\mathrm{N}$-terminal kinase activation and inhibited apoptosis induced by oxidative stress [51]. Resveratrol, curcumin and salvianolic acid derived from traditional Chinese herb Salvia miltiorrhiza protected neurons in vivo and in vitro by activation of PI3K/Akt pathways. Hesperetin activated Akt and downregulated proapoptotic proteins, such as apoptosis signal-regulating kinase 1 (ASK1), Bad, caspase-9 and -3 in hippocampus neurons [52]. Akt phosphorylates longevity transcription factor Forkhead box O3 (FOXO3) and inhibitors of NF- $\mathrm{KB}(\mathrm{I} \kappa \mathrm{B})$, and activates NF- $\mathrm{B}$ to upregulate the expression of prosurvival IAP (inhibitor of apoptosis protein) and Bcl-2 protein family. EGCG, hesperetin, resveratrol, curcumin and caffeic acid activated PI3K/Akt- or ERK-CREB-BDNF cascade in vivo and in vitro, leading to protect neurons, promote memory and reverse depression-like behavior [53]. Resveratrol attenuated $\mathrm{MPP}^{+}$-induced mitochondrial dysfunction and apoptosis through inactivation of GSK-3 $\beta$ by phosphorylated Akt [54].

Polyphenols activate PKC, a member of a serine-threonine kinase superfamily, which regulates cell survival, apoptosis, neuronal differentiation, immune responses, learning and memory [55]. In the striatum of MPTP-treated mice, EGCG upregulated PKC $\alpha$ and PKCE, prevented deletion of PKC and degraded apoptogenic Bad by the UPS [56]. EGCG and resveratrol activated PKC $\gamma$ and increased neuronal interconnection in the rat hippocampus [57].

Polyphenols regulate the expression of antioxidant enzymes through activation of Keep1/Nrf2/antioxidant response element (ARE) pathway. Keap-1 binds to transcription regulator nuclear factor erythroid-derived 2-related factor 2 (Nrf2) and prevents the signal activation. Astaxanthin, lutein, lycopene, baicalein, carnosic acid, carnosol and hydroxytyrosol have been reported to dissociate Keap-1 from the complex with $\mathrm{Nrf2}$, induce translocation of phosphorylated Nrf2 into the nuclei and binding to ARE in the regulatory region of the target genes, and enhance expression of Phase II enzymes, such as glutathione $S$-transferases, glutathione peroxidase, glutathione reductase, superoxide dismutase and NQO1 [58]. Keap1/Nrf2/ARE pathway is activated by PKC $\alpha$ and PI3K/Akt signal pathways, and contributes the antioxidant, anti-inflammatory and neuroprotective functions of polyphenols.

\section{Phytochemicals induce prosurvival NTFs \& NTF receptors}

Polyphenols have been reported to increase BDNF, GDNF and other NTFs in humans and animal and cellular models, and ameliorate neurochemical and behavioral changes. In humans, green tea catechin and coffee fruit extract increased plasma BDNF levels [59,60]. Ginkgo biloba supplement was reported to enhance serum BDNF and aerobic performance in physically active men [61], and curcumin to increase serum BDNF levels and ameliorate the syndrome in women with premenstrual syndrome [62]. In animal models of aging, depression, AD, PD, stroke and brain ischemia, flavonoids (EGCG, hesperidin, 3,5,6,7,8,3',4'-heptamethoxyflavone, naringen, ginkgolide Rd), curcumin, resveratrol, astaxanthin and piperine (a major alkaloid of black pepper) increased BDNF (Figure 3). In primarily and clonally cultured neurons and astrocytes, flavonoids, resveratrol, rosmarinic acid, and oleuropein (a polyphenol constituent of olive oil) induced BDNF and GDNF expression and showed neuroprotection via ERK/cAMP response element-binding protein (CREBS), or PI3K/Akt pathways. Naringen, catalpol derived from herb Rehmannia glutinosa, smilagenin (a sapogenin from Rhizoma anemoarrhence) and harpagoside purified from Scrophularia ningpoensis increased expression of GDNF in animal models of PD [63,64]. In general, BDNF is induced by flavonoids in vivo and in vitro, whereas GDNF is mostly by nonflavonoid phytochemicals like curcumin, resveratrol and catalpol [11].

Polyphenols increase Trk expression and neurogenesis to show neuroprotection. Olive polyphenols increased TrkB and TrkA in the hippocampus, but did not in the striatum and frontal cortex, and presented antidepressantlike effects in animal models of depression [65]. EGCG increased BDNF, TrkA and TrkB expression, and prevented the pathological changes in a mouse model of AD [66]. Deoxygedunin and ethanol extracts from Hemerocallis citrina, and nobiletin isolated from citrus fruits increase TrkB and BDNF expression in mice [67] and promoted hippocampal neurogenesis in a rat model of depression [68]. 


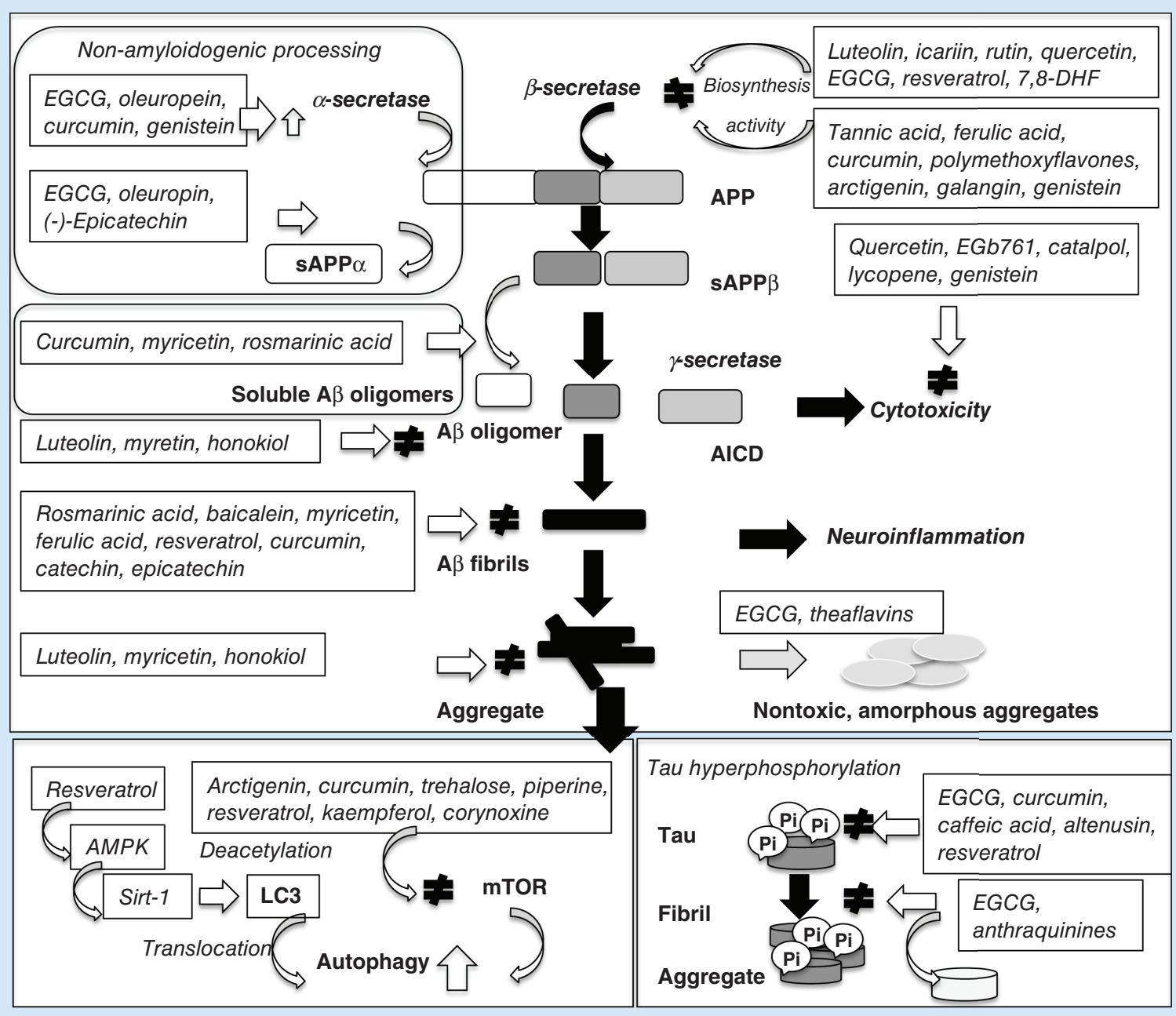

Figure 4. Phytochemicals modulate $\mathbf{A} \beta$ pathology and tau hyperphosphorylation. Phytochemicals increase $\alpha$-secretase activity or inhibit $\beta$-secretase activity, and prevent the production of toxic oligomer and fibril. Curcumin and other polyphenols change the mature $A \beta$ aggregates into nontoxic molecules. Phytochemicals inhibit mTOR and promote autophagy, whereas resveratrol activates 5'-AMP-activated protein kinase-Sirt-1 pathway, deactylates and translocates LC3 and transcriptionally increases autophagic efflux. Polyphenols also inhibit the synthesis, oligomer formation and aggregation of hyperphosphorylated tau and reduce aggregates in vivo and in vitro.

\section{Phytochemicals directly prevent the formation of disease-specific protein aggregates}

Accumulation of senile plaques and neurofibrillary tangle is the major histopathological finding in $\mathrm{AD}$, and that of Lewy bodies and neurites in PD. Abnormal-mutated and -modified protein is aggregated through oligomerization and polymerization, changes in the secondary structure ( $\beta$-sheet formation), formation of insoluble aggregate structure and dysfunction of the proteolytic pathways. Recently, the process, rather than the end product, is considered to decide the neurotoxicity, and the therapeutic strategy should mainly aim to deplete toxic oligomers and fibrils, and aggregation intermediates.

Phytochemicals were found to inhibit the synthesis of amyloidogenic monomers and formation of oligomeric and fibrillar aggregates, promote nontoxic aggregate formation, and activate the proteolytic systems to ameliorate neuronal dysfunction caused by $A \beta$ (Figure 4) [69,70]. Amyloidogenic $A \beta$ peptides with 40-42 amino acids are produced by sequential cleavage of APP by $\beta$-secretase (BACE1) and $\gamma$-secretase. Tannic acid (a polyphenol), ferulic acid, polymethoxyflavones (nobiletin, tangeretin, sinensetin), genistein and galangin have been shown to inhibit $\beta$-secretase and improve behavioral impairment in animal models of $\mathrm{AD}$ [71,72]. Luteolin, icariin, rutin, quercetin, EGCG, resveratrol, curcumin and 7,8-dihydroxyflavine reduced the expression of $\beta$-secretase. EGCG, 
oleuropein, genistein and curcumin promoted APP cleavage by $\alpha$-secretase into nontoxic $N$-terminal product named as soluble APP $\alpha$ and $C$-terminal fragment $\alpha$ [73].

Curcumin, myricetin and rosmarinic acid produced soluble $A \beta$ oligomer and profibrillar species and decreased the toxic oligomers and fibrils [74,75]. EGCG was found to interact with the hydrophobic sequences of amino acids 14-24 and 27-37 of insoluble $A \beta$, break $\beta$-sheet motif, prevent formation of fibrils and convert large mature $\mathrm{A} \beta$ and $\alpha$ Syn fibrils into nontoxic, smaller, amorphous protein aggregate [76]. Luteolin, myricetin and honokiol (the Magnolia-derived lignan) bound to the hydrophobic region of the amyloid pentamer and inhibited $A \beta$ aggregation [77]. Theaflavins stimulated the assembly of $A \beta$ and $\alpha$ Syn into nontoxic spherical aggregates that were incompetent in seeding amyloid formation, and remodeled $A \beta$ fibrils into nontoxic aggregates [78].

Tau is a normal, unfolded, highly soluble protein, and the imbalance between kinases and phosphatases produces hyperphosphorylated tau. Tau hyperphosphorylation changes the conformation into aggregate-prone species: conversion of the monomer to oligomer and aggregation into pair helical filaments, resulting in production of neurofibrillary tangles. The immediate tau oligomer is a most toxic form and causes synaptic impairment in AD. EGCG, resveratrol, curcumin, caffeic acid and altenusin inhibited tau hyperphosphorylation [79,80]. EGCG prevented aggregation of tau into toxic oligomers [81], and anthraquinones (emodin, daunorubicin) inhibited tau aggregation and dissolved paired helical filaments in vitro and in cells [82].

Aggregation of presynaptic $\alpha$ Syn has been proposed as a pathogenic factor in ' $\alpha$-synucleinopathies' including PD. $\alpha$ Syn aggregation and fibrillization are induced by the increase in, missense mutations (A30P, A53T, E46K), oxidative stress, phosphorylation, metal ions $\left(\mathrm{Fe}^{3+}, \mathrm{Cu}^{2+}\right)$ and interaction with phospholipid membrane. As in the case with $A \beta$ and tau, intermediate formation of the toxic oligomers is most critical for the $\alpha$ Syn neurotoxicity. EGCG, baicalein, myricetin, kaempferol, theaflavins, curcumin, nordihydroguaiaretic acid, rosmarinic acid and ferulic acid inhibited $\alpha$ Syn oligomer formation and assembly into aggregates [83,84]. EGCG converted mature $\alpha$ Syn fibrils into nontoxic, smaller, amorphous aggregates [85]. Curcumin was reported to bind to preformed toxic oligomer and fibrils, and alter the hydrophobic surface into more soluble species [86].

Protein aggregation is caused also by dysfunction of the cellular proteolytic systems, the UPS and the ALP, as discussed in Box 3. The UPS targets the unfolded ubiquitinated protein and the dysfunction results in accumulation of misfolded protein in PD, but the role of the USP in the pathogenesis has not been fully established. The ALP targets disease-specific abnormal protein aggregates, including A $\beta$, $\alpha$ Syn, $N$-terminal fragments of mutant Huntingtin in Huntington's disease and mutant TDP43 in amyotrophic lateral sclerosis (aggrephagy) and damaged mitochondria (mitophagy) [87]. Autophagy activation has been shown to remove aggregate-prone proteins, regulate axon homeostasis and neurogenesis, and modify the pathogenesis of $\mathrm{PD}, \mathrm{AD}$ and other neurodegenerative disorders [88].

The mTOR/ULK1 is a major negative regulator of autophagy, and the Beclin-1 (ATG6) is a positive regulator. Phytochemicals induce autophagy and show neuroprotection through mTOR-dependent or -independent mechanisms. In mouse models of PD, resveratrol prevented MPTP-induced neurotoxicity via autophagic degradation of $\alpha$ Syn and efflux, which was mediated by Sirt-1-dependent deacetylation of LC3 (MAP1LC3A) and its translocation from nucleus to cytoplasm [89]. Curcumin protected PC12 cells against A53T $\alpha$ Syn-induced cytotoxicity by downregulation of mTOR/p70S6K (p70-S6 kinase) signaling and activation of autophagy [90]. In APP/PS1 double transgenic mice, curcumin activated autophagy and protected neurons via downregulation of PI3K/Akt/mTOR signaling pathway [91]. Oleuropein aglycone induced autophagy by activation of AMPK/mTOR signal pathway, decreased $A \beta$ aggregation and ameliorated cognition impairment [92]. Resveratrol, curcumin and EGCG inhibited AMPK and initiated mTOR-mediated autophagic degradation in cellular models of PD and AD [93,94].

\section{Conclusion}

Phytochemicals protect neuron by targeting multiple pathogenic factors of neurodegenerative disorders. Epidemiological studies have presented that dietary habits promote health and longevity, preserve cognition and motor function, and prevent depressive disorders. Intervention with food-derived compounds has been proposed as the therapeutic strategy for age-dependent neurodegeneration. However, the beneficial effects of phytochemicals in the clinical studies have not been fully confirmed. Bioavailability of the food-derived compounds is limited by the rapid metabolism, insufficient permeability across the BBB, and decreased bioavailability and stability in the brain [95]. Lipophilic flavonoids, alkaloids and terpenes can be transferred into the brain across the BBB and have high bioavailability and good affinity to receptors in the brain [96]. Development of new neuroprotective compounds is expected among these phytochemicals and the derivatives. In order to increase bioavailability and pharmacological 
Box 3. Proteolysis systems to control protein quantity in neurons.

The ubiquitin-proteasome system and ALP are two major proteolytic systems in neurons and the dysfunction causes formation of proteinous inclusion body in neurodegenerative disorders.

The ubiquitin-proteasome system maintains rapid protein turnover in the cytosol and nuclei by removal of abnormal, misfolded protein and regulates synaptic proteins. The targeted protein is covalently modified with branched polyubiquitin chains and degraded in 265 proteasome complex.

The ALP recycles nutrients during starvation and protects neurons by degradation of long-lived insoluble inclusions and damaged mitochondria. Knockout of neuron-specific autophagic genes, such as atg5, atg7 and beclin1 in mice, inhibits autophagy, and progressive motor deficit is developed with inclusion body accumulation.

The ALP is divided into chaperone-mediated autophagy, microautophagy and macroautophagy.

Chaperone-mediated autophagy is a process in which chaperone $\mathrm{Hsc70}$ targets the substrate with a

lysyl-phenylalanyl-phenylalanyl-argininyl-glutamyl (KFFRQ) motif to the lysosome membrane. Microautophagy involves internalized cytosolic cargo and fuses directly with lysosome. Macroautophagy (here referred to as autophagy) degrades cytosolic protein aggregates and organelles. Superfluous or dysfunctional organelles are sequestered by phagophore, which matures in the double-membrane autophagosomes, and fuses with lysosomes, where the cargo materials are degraded.

Autophagy is negatively regulated by mTOR kinase, and mTOR suppresses autophagy through direct interaction with the ULLK1-Atg13-FIP200 complex. Under starvation mTOR-mediated phosphorylation of Atg12 and ULK1 is inhibited and autophagy is activated.

Autophagosme formation is initiated by Vps34 of autophagy-regulating macromolecular complex (PI3K complex), containing Beclin-1. Beclin-1 regulates autophagy positively with AMBRA1, UVRAG and Bif-1, or negatively with $\mathrm{BCl}-2$ and $\mathrm{Bcl}-\mathrm{xl}$.

Autophagy is regulated post-transcriptionally by phosphorylation, ubiquitination and acetylation directly or indirectly by modulating regulators localized at upstream of the Atg proteins, in order to adapt to environmental stress. Serum starvation activates GSK3 and acetyltransfease TIP60, which directly acetylates ULK1 and initiates autophagy. Sirt 1 and EP300/p300 acetylate Atg5, Atg7, Atg8 and Atg12, and induce autophagy.

Protein products of genes associated with PD, parkin (PARK2, E3 ubiquitin protein ligase) and PINK1 regulate mitophagy by the ALP. Loss of DJ-1 enhances autophagic efflux. G2019S mutation of LRRK 2 increases autophagic vacuole and autophagy-dependent shortening of neurites, and LRRK 2 interacts with Beclin-1 and regulates autophagy.

ALP: Autophagy-lysosome pathway.

formulation, several delivery systems have been proposed, including nanoparticles, liposomes, complexes with phospholipids and amphiphilic polymers and conjugation with amino acids and glycosides [97].

In cellular models, phytochemicals suppress or promote the mitochondrial permeabilization and apoptosis dependently on the concentrations, suggesting that 'therapeutic window' of food-derived compounds is too narrow to present reproducible results in clinical trials. The effects of diet and food-derived compounds should vary also among individuals with different genetic background, metabolic profile, age and environmental exposure. These facts suggest that the results obtained by epidemiological study should be carefully evaluated. Highly sensitive analytical methods for pharmacokinetic study are required to identify the potent bioactive compounds and determine the effective concentration in the targeted brain regions. In addition, the quantitative evaluation of the clinical benefits and effects on disease progression has not been established, and more sensitive in vivo quantitative methods of the survival or death and function of distinct neurons are still required.

BDNF and GDNF have been applied to patients with PD, depressive disorders and cognition decline, and the BBB-permeable phytochemicals might be useful as a surrogate delivery tool by stimulating the de novo synthesis of NTFs in the brain. BDNF and GDNF have been reported to be selectively induced by flavonoids and nonflavonoids in glial cells. It may be relevant with the results that flavonoids improve memory, cognition and depression, whereas resveratrol and curcumin ameliorate neuronal dysfunction and promote survival in animal models. Induction of distinct NTF family should be systematically investigated to establish the structure linking to increase the specified NTF synthesis.

Recently, new neuroprotective multitargeted MAO-B inhibitors have been derived from the basic structure of phytochemicals. Pyrazoline analogs of MAO inhibitors were synthesized from sasamol, curcumin and flavones [98,99]. Coumarin is found in many plants and characterized by 1,2-benzopyrone or benzopyran-2-one groups, and various drugs, inducing warfarin, acenovoumarol and phenprocoumarin have been developed. Dual acetylcholinesterase$\mathrm{MAO}-\mathrm{B}$ inhibitors, such as tacrine-coumarin hybrids, were reported as new drug candidates for $\mathrm{AD}$ [100]. Tacrine was 
the first AChE inhibitor on the market and coumarins are inhibitors of $\mathrm{MAO}$ and AChE activity. AChE inhibitors not only increase cholinergic transmission, but also interfere with the synthesis, deposition and aggregation of $A \beta$. Homoisoflavonoid Mannich base derivatives were found to have high affinity toward A $\beta$ aggregates and MAO-B inhibitory activities, and multifunctional agents with AChE inhibition, including tacrine derivatives, have been developed [101,102]. By in silico screening for AChE inhibitors, plant benzyl alkaloids (berberastine, berberine, yohimbine, sanguinarine) and naringenin were confirmed to inhibit AChE, suggesting finding of novel anti-AD drugs [103].

The antidepressant and anticancer activities of phytochemicals have been intensively investigated, but cannot be discussed in details in this review. Depressive disorders are caused not only by the deregulation of monoamine neurotransmitter systems, but also by impaired development and function of neural architecture by the interaction between genetic and environmental factors [104].

Diet, curcumin, ferulic acid, hypericum and resveratrol presented the preventive and therapeutic activities in depressive disorders by induction of BDNF expression and promotion of adult neurogenesis in the hippocampus [105]. Phytochemicals have inhibited or promoted mitochondrial apoptosis signaling to show neuroprotective or anticancer activities through regulation of mitochondrial membrane permeability [106]. Our recently reported analytical method for the apoptosis signaling at the mitochondrial membrane [9] can be applied to screen the structure required for the inhibitory or promoting potency of phytochemicals. These prominent functions of phytochemicals should be further studied for development of novel series of antidepressants and anxiolytics and also anticancer therapeutic agents.

\section{Future perspective}

Phytochemicals and the derivatives are applied as preventive and therapeutic compounds in patients with neuropsychiatric diseases, including AD, PD and other neurodegenerative disorders, and depression. Using more advanced brain image analyses, the effects on function of specified neurons in the brain regions can be followed quantitatively in vivo. More sensitive and exact analyses of phytochemical metabolites supply the on-time information of the distribution, metabolism and bioactivity of phytochemicals in the brain. These results are applied to develop new neuroprotective compounds with more stable bioavailability and higher neuroprotective potency by induction of in situ biosynthesis of NTFs, Bcl-2 protein family and antioxidant molecules. Molecular mechanisms underlying antiamyloidogenic effects of phytochemicals are clarified in more detail, and phytochemical derivatives are applied to prevent formation of toxic $A \beta$ and $\alpha$ Syn oligomers in the brain of patients with $A D$ and $P D$. Instead of once proposed antibody therapy, phytochemicals are applied to covert mature protein aggregates into soluble nontoxic forms. From the BBB-permeable phytochemicals, new multifunctional compounds will be synthesized with inhibitory potency to MAO and AChE in order to increase monoamine transmitters and acetylcholine in addition to the neuroprotective activity for the therapy of $\mathrm{AD}$ and $\mathrm{PD}$.

\section{Executive summary}

Targets for neuroprotection by phytochemicals

- Phytochemicals protect neuronal cells by modification of etiopathogenic factors in neurodegenerative disorders: oxidative stress, mitochondrial dysfunction, activated mitochondrial apoptosis cascades, deficits of neurotrophic factors, and protein aggregation and accumulation.

- Phytochemicals modify cellular signal pathways to induce expression of neuroprotective genes.

Phytochemicals: the classification, structure \& antioxidant \& anti-inflammatory functions

- Main neuroprotective phytochemicals are polyphenols including flavonoids and nonflavonoids, such as phenolic acids, stilbene, curcumin, astaxanthin and sesame lignans.

- Flavonoids are potent antioxidants in vitro and can scavenge reactive oxygen species (ROS)/reactive nitrogen species (RNS), and also chelate ferric and copper ions. The antioxidant function depends on the hydroxyl groups on the aromatic $A$ and $B$ rings, and unsaturation in the $C$ ring.

- Polyphenols increase expression of ROS-scavenging enzymes and inhibit that of ROS-producing enzymes.

- Flavonoids exhibit anti-inflammation activity by the antioxidant activity and modulation of signal transduction for synthesis of proinflammatory cytokines.

Phytochemicals protect mitochondrial function \& increase biogenesis

- Phytochemicals protect mitochondrial function against mitochondrial toxicity of accumulated pathogenic $A \beta$ and aSyn.

- Phytochemicals increase mitochondrial biogenesis and control their quality by fission-fusin and cleavage of damaged mitochondria in the autophagy-lysosome system (mitophagy). 
Phytochemicals intervene mitochondrial cell death cascade

- Phytochemicals directly regulate mitochondrial membrane permeabilization, the initial step to apoptotic process.

- In dependence of the lypophilicity and redox potency, phytochemicals prevent or enhance step-wise opening of pore at the inner and outer mitochondrial membranes.

- Curcumin prevents oxidative modification of the critical thiol residues of components of mitochondrial permeability transition pore and induction of apoptosis.

- Flavonoids increase expression of antiapoptotic $\mathrm{Bcl}-2$ protein family and prevent the mitochondrial permeability transition pore opening.

Phytochemicals present neurotrophic factor-like activity by binding to neurotrophic factor receptors \& activating signal pathways for neuroprotection

- Neurotrophic factors (NTFs), especially brain-derived neurotrophic factor (BDNF) and glial cell line-derived neurotrophic factor (GDNF), regulate function and survival of neurons, and phytochemicals have been confirmed to function like NTFs in the brain.

- 7,8-DHF and the analogs bind to tropomycin-related kinase B (TrkB), the BDNF receptor and activate downstream signal pathways.

- The binding of polyphenols to other receptors, including GABA, nicotine acetylcholine, serotonin, $\kappa$-opioid receptor and proteins, such as monoamine oxidase, mediate survival signaling for neuroprotection.

Phytochemicals modulate cellular signal pathways for neuroprotection

- Phytochemicals activate prosurvival MAPK pathways, including PI3K/Akt and PKC, and preserve cellular function and synaptic plasticity for neuroprotection.

- Polyphenols activate Keap1/Nrf2/antioxidant response element pathway to induce antioxidant Phase II enzymes.

Phytochemicals induce prosurvival NTFs \& NTF receptors

- In human, catechin, Ginkgo biloba supplement and curcumin increased serum BDNF.

- In animal models and culture neuronal and glial cells, flavonoids increase BDNF and glial cell line-derived neurotrophic factor by activation of ERK/CREBS or PI3K/Akt pathways.

- Polyphenols increase TrkB and TrkA expression and neurogenesis to present neuroprotection and antidepressant activity.

Phytochemicals directly prevent the formation of disease-specific protein aggregates

- Polyphenols inhibit the activity of $\gamma$-secretase and downregulate the expression to prevent the synthesis of amyloidogenic monomer of $A \beta$..

- Flavonoids and curcumin enhance $\alpha$-secretase activity and promote amyloid precursor protein cleavage into nontoxic fragments.

- Curcumin directly changes the $A \beta$ conformation from oligomers and fibrils into soluble ones.

- EGCG breaks $\beta$-sheet motif of insoluble $A \beta$ and converts large mature fibrils into nontoxic smaller protein aggregates.

- EGCG, resveratrol and curcumin inhibit tau hyperphosphorylation to suppress formation of toxic oligomers.

- Flavonoids inhibit production of toxic $\alpha$ Syn oligomers, and EGCG converts mature $\alpha$ Syn fibrils into nontoxic aggregates.

- Phytochemicals induce autophagy, prevent accumulation of damaged proteins and organelle, and protect cells.

Financial \& competing interests disclosure

The authors' research on neuroprotection by food-derived bioactive compounds is supported by the Grants-in-Aids for Scientific Research, No. 18Kk07430, Furukawa Academic Research Promotion Foundation, and The Tojuro lijima Foundation for Food Science and Technology (W Maruyama). The authors have no other relevant affiliations or financial involvement with any organization or entity with a financial interest in or financial conflict with the subject matter or materials discussed in the manuscript apart from those disclosed.

No writing assistance was utilized in the production of this manuscript.

\section{Open access}

This work is licensed under the Creative Commons Attribution-NonCommercial-NoDerivatives 4.0 Unported License. To view a copy of this license, visit http://creativecommons.org/licenses/by-nc-nd/4.0/ 


\section{References}

Papers of special note have been highlighted as: $\bullet$ of interest; $\bullet \bullet$ of considerable interest

1. Schapira AHV, Olanow CW. Neuroprotection in Parkinson disease. Mysteries, myths, and misconceptions. JAMA 291(3), 358-364 (2004).

2. Sanchez-Villegas A, Delgado-Rodriguez M, Alonso A et al. Association of the Mediterranean dietary pattern with the incidence of depression. Arch. Gen. Psychiatry 66(10), 1090-1098 (2009).

3. Singh B, Parsaik AK, Mielke MM et al. Association of Mediterranean diet with mild cognitive impairment and Alzheimer's disease: a systemic review and meta-analysis. J. Alzheimers Dis. 39(2), 271-282 (2014).

- A systemic review from 664 studies on the effects of diet on cognitive impairment in Alzheimer's disease and mild cognitive impairment.

4. Rabassa M, Cherubin A, Zamora-Ros R et al. Low level of a urinary biomarker of dietary polyphenol are associated with substantial cognitive decline over a 3-year period in older adults: The Invecchiare in Chianti Study. J. Am. Geriatr. Soc. 63(5), 938-946 (2015).

5. Son JX, Sze SCW, Ng TB et al. Anti-Parkinsonian drug discovery from herbal medicines: what have we got from neurotoxic models? J. Ethanopharm. 139(3), 698-711 (2012).

- Presents the neuroprotective activity and intracellular target sites of herbal samples and the major components in vitro and in vivo models of Parkinson's and Alzheimer's diseases.

6. Mancuso C, Sciliano R, Barone E, Preziosi P. Natural substances and Alzheimer's disease: from preclinical studies to evidence based medicine. Biochim. Biophys. Acta 1822(5), 616-624 (2012).

- Presents the neuroprotective activity and intracellular target sites of herbal samples and the major components in vitro and in vivo models of Parkinson's and Alzheimer's diseases.

7. Macready AL, Kennedy OB, Ellis JA et al. Flavonoids and cognitive function: a review of human randomized controlled trial studies and recommendations for future studies. Gene Nutr. 4(4), 227-242 (2009).

8. Huhn S, Beyer F, Zhang R et al. Effects of resveratrol on memory performance, hippocampus connectivity and microstructure in older adults - a randomized control trial. Neuroimage 174, 177-190 (2018).

-• Could present only nonsignificant positive effects on memory, but the methods and results are suggestive for evaluation of the effects in future clinical trials.

9. Wu Y, Shamoto-Nagai M, Maruyama W, Osawa T, Naoi M. Phytochemicals prevent mitochondrial membrane permeability and protect SH-SY5Y cells against apoptosis induced by PK11195, a ligand for outer membrane translocator protein. J. Neural Transm. 124(1), 89-98 (2017).

10. Moosavi F, Hosseni R, Saso L, Firuz O. Modulation of neurotrophic signaling pathways by polyphenols. Drug Design Develop. Ther. 10, 23-42 (2016)

- Presents the neurotrophic factor-like function of phytochemicals in the brain and the cellular signal pathways associated with neuroprotection.

11. Naoi M, Inaba-Hasegawa K, Shamoto-Nagai M, Maruyama W. Neurotrophic function of phytochemicals for neuroprotection in aging and neurodegenerative disorders: modulation of intracellular signaling and gene expression. J. Neural Transm. 124(12), 1515-1527 (2017).

12. Wang ZY, Liu JY, Yang CB et al. Neuroprotective natural products for the treatment of Parkinson's disease by targeting the autophagy-lysosome pathway: a systematic review. Phytochem. Res. 31(8), 1119-1127 (2017).

13. Dias V, Junn E, Mouradian MM. The role of oxidative stress in Parkinson's disease. J. Parkinsons Dis. 3(4), 461-491 (2013).

14. Wang X, Wang W, Li L, Perry et al. Oxidative stress and mitochondrial dysfunction in Alzheimer's disease. Biochim. Biophys. Acta 1842(8), 1240-1247 (2014).

15. Chu KO, Chan S, Pang CP, Wang CC. Pro-oxidative and antioxidative controls and signaling modification of polyphenolic phytochemicals: contribution to health promotion and disease prevention? J. Agri. Food Chem. 62(8), 4026-4038 (2014),

- Discusses the ambivalent function of phytochemicals either as anti- or pro-oxidants.

16. Liang J, Tian YX, Yang F, Zhang JP, Skibsted LH. Antioxidant synergism between carotenoids in membrane. Astaxanthin as a radical transfer bridge. Food Chem. 115, 1437-1442 (2009).

17. Echeverry C, Arrendondo G, Abin-Carriquiry JA et al. Pretreatment with natural flavones and neuronal survival after oxidative stress: a structure-activity relationship study. J. Agri. Food Chem. 58(4), 2111-2115 (2010).

18. Hirsch EC, Hunot S. Neuroinflammation in Parkinson's disease: a target for neuroprotection? Lancet Neurol. 8(4), 382-397 (2009).

19. Rahman I, Biswas SK, Kirkham PA. Regulation of inflammation and redox signaling by dietary polyphenols. Biochem. Pharmacol. 72(11), 1439-1452 (2006).

20. Ray B, Lahiri DK. Neuroinflammation in Alzheimer's disease: different molecular targets and potential therapeutic agents including curcumin. Curr. Opin. Pharmacol. 9(4), 434-444 (2009).

21. Mattson MP, Gleichmann M, Cheng A. Mitochondria in neuroplasticity and neurological disorders. Neuron 60(5), 748-766 (2008). 
22. Schapira AH. Mitochondria in the aetiology and pathogenesis of Parkinson's disease. Lancet Neurol. 7(1), 97-109 (2008).

23. Forbes-Hernandez TY, Giampieri F, Gasparrini M et al. The effects of bioactive compounds from plant foods on mitochondrial function: a focus on apoptotic mechanisms. Food Chem. Toxicol. 68, 154-182 (2014).

The effects of phytochemicals on mitochondrial apoptosis machinery, protection of the function and association of neuroprotective signal pathways were reviewed.

24. Valenti D, De Rasmo D, Signorile A et al. Epigallocatechin-3-gallate prevents oxidative phosphorylation deficit and promotes mitochondrial biogenesis in human cells from subjects with Down syndrome. Biochim. Biophys. Acta 1832(4), 542-552 (2013).

25. Parrado-Fernandez C, Sandebring-Matton A, Rodriguez-Rodringuez P, Aarsland D, Cedazo-Minguez A. Anthocyanins protect from complex I and APPswe mutation through modulation of the mitochondrial fission/fusion pathways. Biochim. Biophys. Acta 1862(11), 2110-2118 (2016).

26. Jo DS, Shin DW, Park SJ et al. Attenuation of A $\beta$ toxicity by promotion of mitochondrial fusion in neuroblastoma cells by liquiritigenin. Arch. Pharm. Res. 39(8), 1137-1143 (2016).

27. Peng $\mathrm{K}$, Tao $\mathrm{Y}$, Zhang J et al. Resveratrol regulates mitochondrial biogenesis and fission/fusion to attenuate rotenone-induced neurotoxicity. Oxid. Med. Cell. Longev. 2016, 670562 (2016).

28. de Oliveira MR, Nabavi SF, Manyi A et al. Resveratrol and mitochondria: from triggering the intrinsic apoptotic pathway to inducing biogenesis, a mechanistic view. Biochim. Biophys. Acta 1860(4), 727-745 (2016).

29. Sandoval-Acuria C, Ferreira J, Speisky H. Polyphenols and mitochondria: an update on their increasingly emerging ROS-scavenging independent actions. Arch. Biochem. Biophys 559, 75-90 (2014).

-. Summarizes recent results of the effects of polyphenols on mitochondrial function and apoptosis, which were independent of reactive oxygen species-scavenging functions.

30. Nieman DC, Williams AS, Shanely RA et al. Quercetin's influence on exercise performance and muscle mitochondrial biogenesis. Med. Sci. Sports Exerc. 42(2), 338-345 (2010).

31. Camilleri A, Zarb C, Caruana M et al. Mitochondrial membrane permeabilisation by amyloid aggregates and protection by polyphenols. Biochim. Biophys. Acta 1828(11), 2532-2543 (2013).

32. Gorlach S, Fichna J, Lewandowska U. Polyphenols as mitochondria-targeted anticancer drugs. Cancer Lett. 366(2), 141-149 (2015).

33. Salvi M, Brunati AM, Clari G, Toninello A. Interaction of genistein with the mitochondrial electron transport chain results in opening of the membrane transition pore. Biochim. Biophys. Acta 1556(2-3), 187-196 (2002).

34. De Marchi U, Biasutto L, Garbisa SP, Toninello A, Zorratti M. Quercetin can act either as an inhibitor or an inducer of the mitochondrial permeability transition pore: a demonstration of the ambivalent redox character of polyphenols. Biochim. Biophys. Acta 1787(12), 1425-1432 (2009).

- Presents the mitochondrial membrane permeabilization by polyphenols and the anticancer activity.

35. Zhang H, Ozbay F, Lappalainen J et al. Brain derived neurotrophic factor gene (BDNF) variants and Alzheimer's disease, affective disorders, posttraumatic stress disorders, schizophrenia and substrate dependence. Am. J. Med. Genet. B Neuropsychiatr. Genet. 141B(4), 387-393 (2006).

36. Hegarty SV, Lee DJ, O'Keeffe GW, Sullivan AM. Effects of intracerebral neurotrophic factor application on motor symptoms in Parkinson's disease: a systematic review and meta-analysis. Parkinsonism Relat. Disord. 38, 19-25 (2017).

37. Youdim KA, Shukitt-Hale B, Joseph JA. Flavonoids and the brain: interactions at the blood-brain barrier and their physiological effects on the central nervous system. Free Rad. Biol. Med. 37(11), 1683-1693 (2004).

38. Liu C, Chan CB, Ye K. 7, 8-Dihydroxyflavone, a small molecular TrkB agonist, is useful for treating various BDNF-implicated human disorders. Transl. Neurodegenerat. 5, 2 (2016).

39. Hwang SL, Yen GC. Effects of hesperetin against oxidative stress via ER- and TrkA-mediated actions in PC12 cells. J. Agri. Food Chem. 59(10), 5779-5785 (2011).

40. Al-Gayyar MM, Matragoon S, Pillai BA et al. Epicatechin blocks pro-nerve growth factor (proNGF)-mediated retinal degeneration via inhibition of p75 neurotrophic factor expression in a rat model of diabetes. Diabetologia 54(3), 669-680 (2011).

41. Han YS, Bastianetto S, Dumont Y, Quirion R. Specific plasma membrane binding sites for polyphenols, including resveratrol, in the rat brain. J. Pharmacol. Exp. Ther. 318(1), 228-245 (2006).

42. Umeda D, Yano S, Yamada K, Tachibana H. Green tea polyphenol epigallocatechin-3-gallate signaling pathway through 67-kDa laminin receptor. J. Biol. Chem. 283(6), 3050-3058 (2008).

43. Naoi M, Maruyama W, Shamoto-Nagai M. Type A and B monoamine oxidase distinctly modulate signal transduction pathway and gene expression to regulate brain function and survival of neurons. J. Neural Trans. 125(11), 1635-1650 (2018).

- Novel functions of monoamine oxidases as a modulator of cellular signal pathways for neuroprotection were discussed.

44. Carradori S, Gidaro MC, Petzer A et al. Inhibition of human monoamine oxidase: biological and molecular modeling studies on selected natural flavonoids. J. Agric. Food Chem. 64(47), 9004-9011 (2016). 
45. Ali Shah S, Ullah I, Lee HY, Kim MO. Anthocyanins protect against ethanol-induced neuronal apoptosis via $\mathrm{GABA}_{\mathrm{B} 1}$ receptors intracellular signaling in prenatal rat hippocampal neurons. Mol. Neurobiol. 48(1), 257-269 (2013).

46. Dai J, Chen L, Qiu YM et al. Activations of GABAergic signaling, HSP70 and MAPK cascades are involved in baicalin's neuroprotection against gerbil ischemia/reperfusion injury. Br. Res. Bull. 90, 1-9 (2013).

47. Zhang $\mathrm{X}$, Wu M, Lu F et al. Involvement of $\alpha 7 \mathrm{nAChR}$ signaling cascade in epigallocatechin gallate suppression $\beta$-amyloid-induced apoptotic cortical neuronal insults. Mol. Neurobiol. 49(1), 66-77 (2014).

48. Xu Y, Ku B, Cui L et al. Curcumin reverses impaired hippocampal neurogenesis and increases serotonin receptor $1 \mathrm{~A}$ and brain-derived neurotrophic factor expression in chronically stressed rats. Br. Res. 1162, 9-18 (2007).

49. Filho CB, Del Fabbro L, de Gomes MG et al. $\kappa$-opioid receptors mediates the antidepressant-like activity of hesperidin in the mouse forced swimming test. Eur. J. Pharmacol. 698(1-3), 286-291 (2013).

50. Xu SL, Bi CW, Choi RE et al. Flavonoids induce the synthesis and secretion of neurotrophic factors in cultured rat astrocytes: a signaling response mediated by estrogen receptor. Evid. Based Complement. Alternat. Med. 2013, 127075 (2013).

51. Hwang SL, Yen GC. Modulation of Akt, JNK, and p38 activation is involved in citrus flavonoid-mediated cytoprotection of PC12 cells. J. Agric. Food Chem. 57(6), 2576-2582 (2009).

52. Vauzour D, Vafeiadou K, Rice-Evans C, Williams RJ, Spencer JPE. Activation of pro-survival Akt and ERK1/2 signalling pathways underlie the anti-apoptotic effects of flavonones in cortical neurons. J. Neurochem. 103(4), 1355-1367 (2007).

53. Zhang L, Fang Y, Xu Y et al. Curcumin improves amyloid $\beta$-peptide (1-42) induced spatial memory deficits through BDNF-ERK signaling pathway. PLoS ONE 10(6), e0131526 (2015).

54. Zeng W, Zhang W, Lu F, Gao L, Gao G. Resveratrol attenuates $\mathrm{MPP}^{+}$-induced mitochondrial dysfunction and cell apoptosis via AKT/GSK-3 $\beta$ pathway in SN4741 cells. Neurosci. Lett. 637, 50-56 (2017).

55. Das J, Ramani R, Suruju MO. Polyphenol compounds and PKC signaling. Biochim. Biophys. Acta 1860(10), 2107-2121 (2016).

56. Kalfon L, Youdim MBH, Mandel SA. Green tea polyphenol (-)-epigallocatechin-3-gallate promotes the rapid protein kinase C-and proteasome-mediated degradation of Bad: implications for neuroprotection. J. Neurochem. 100(4), 992-1002 (2007).

Reports the activation of PKC by polyphenols as neuroprotective signaling pathway.

57. Menard C, Bastianetto S, Quirion E. Neuroprotective effects of resveratrol and epigallocatechin gallate polyphenols are mediated by the activation of protein kinase C $\gamma$. Front. Cell. Neurosci. 7, 281 (2013).

58. Qin S, Deng F, Wu W et al. Baicalein modulates Nrf/Keap1 system in both Keap1-dependent and Keap1-independnt mechanisms. Arch. Biochem. Biophys. 559, 53-61 (2014).

59. Singh BN, Shankar S, Srivastava RK. Green tea catechin, epigallocatechin-3-gallate (EGCG): mechanisms, perspectives and clinical applications. Biochem. Pharmacol. 82(12), 1807-1821 (2011).

60. Neshatdoust S, Saunders C, Castle SM et al. High-flavonoid intake induces cognitive improvement linked to changes in serum brain-derived neurotrophic factor: two randomised, control trials. Nutr. Healthy Aging 4(1), 81-91 (2016).

61. Sadowska-Krepa E, Klapcinska B, Pokora I et al. Effects of 6-week Ginkgo biloba supplementation on aerobic performance, blood pro/antioxidant balance, and serum brain-derived neurotrophic factor in physically active men. Nutrients 9(8), 803 (2017).

62. Fanaei H, Khayat S, Kasaeian A, Javadimehr M. Effect of curcumin on serum brain-derived neurotrophic factor levels in women with premenstrual syndrome: a randomized, double-blind, placebo-controlled trial. Neuropeptides 56, 25-31 (2016).

63. Xu G, Xiong Z, Yong Y et al. Catalpol attenuates MPTP induced neuronal degeneration of nigral-striatal dopaminergic pathway in mice through elevating glial celled neurotrophic factor in striatum. Neuroscience 167(1), 175-184 (2010).

64. Leem E, Nam JH, Jeon MT et al. Naringin protects the nigrostriatal dopaminergic projection through induction of GDNF in a neurotoxin model of Parkinson's disease. J. Nutri. Biochem. 25(7), 801 (2014).

65. De Nicolo S, Tarani L, Ceccanti $\mathrm{M}$ et al. Effects of olive polyphenols administration on nerve growth factor and brain-derived neurotrophic factor in the mouse brain. Nutrition 29(4), 681-687 (2013).

66. Liu M, Chen F, Sha L et al. (-)-Epigallocatechin-3-gallate ameliorates learning and memory deficits by adjusting the balance of TrkA/p75 ${ }^{\mathrm{NTR}}$ signaling in APP/PSI transgenic mice. Mol. Neurobiol. 49(3), 1350-1363 (2014).

67. Jang SW, Liu X, Chan VB et al. Deoxygedunin, an natural product with potent neurotrophic activity in mice. PLoS ONE 5(7), e11528 (2010).

68. Li J, Zhou Y, Liu BB et al. Nobiletin ameliorates the deficits in hippocampal BDNF, TrkB, and synapsin I induced by chronic unpredictable mild stress. Evid. Based Compliment. Alternat. Med. 2013, 359683 (2013).

69. Smid SD, Maag JL, Musgrave IF. Dietary polyphenol-derived protection against neurotoxic $\beta$-amyloid protein: from molecular to clinical. Food Funct. 2(12), 1242-1250 (2012).

70. Bieschke J. Natural compounds may open new routes to treatment of amyloid diseases. Neurotherapeutics 10(3), 429-439 (2013).

-. Presents the molecular mechanisms behind antiamyloidogenic activity of polyphenols. 
71. Mori T, Rezai-Zadeh K, Koyama $\mathrm{N}$ et al. Tannic acid is a natural $\beta$-secretase inhibitor that prevents cognitive impairment and mitigates Alzheimer-like pathology in transgenic mice. J. Biol. Chem. 287(9), 6912-6927 (2012).

72. Mori T, Koyama N, Guillot-Sestier MV, Tan J, Town T. Ferulic acid is nutraceutical $\beta$-secretase modulator that improves behavioral impairment and Alzheimer-like pathology in transgenic mice. PLoS ONE 8(2), e44774 (2013).

73. Kostomori M, Fragkoul A, Sagnou M et al. Oleuropein, an antioxidant polyphenol constituent of olive promotes $\alpha$-secretase cleavage of the amyloid precursor protein (AßPP). Cell. Mol. Neurobiol. 33(1), 147-154 (2013).

74. Ono K, Takamura Y, Yoshoike Y et al. Phenolic compounds prevent amyloid $\beta$-protein oligomerization and synaptic dysfunction by site-specific binding. J. Biol. Chem. 287(16), 14631-14643 (2012).

75. Thapa A, Jett SD, Chi EY. Curcumin attenuates amyloid- $\beta$ aggregate toxicity and modulates amyloid- $\beta$ aggregation pathway. ACS Chem. Neurosci. 7(1), 56-68 (2015).

76. Bieschke J, Russ J, Friedrich RP et al. EGCG remodels mature $\alpha$-synuclein and amyloid fibrils and cellular toxicity. Proc. Natl Acad. Sci. USA 107(17), 7710-7715 (2010).

77. Das S, Stark L, Musgrave IF, Pukala T, Smid SD. Bioactive polyphenol interactions with $\beta$ amyloid: a comparison of binding modelling, effects on fibril and aggregate formation and neuroprotective capacity. Food Funct. 7(2), 1138-1146 (2016).

78. Grelle G, Otto A, Lorenz M et al. Black tea theaflavins inhibit formation of toxic amyloid- $\beta$ and $\alpha$-synuclein fibrils. Biochemistry 50(49), 10624-10637 (2011).

79. Park SY, Kim HS, Cho EK et al. Curcumin protected PC12 cells against $\beta$-amyloid-induced toxicity through the inhibition of oxidative damage and tau hyperphosphorylation. Food Chem. Toxicol. 46(8), 2881-2887 (2008).

80. Chua SW, Comejo A, van Eersei J et al. The polyphenol altenusin inhibits in vitro fibrillization of tau and reduces induced tau pathology in primary neurons. ACS Chem. Neurosci. 8(4), 743-751 (2017).

81. Wobst HJ, Sharma A, Diamond MI, Wanker EE, Bieschke J. The green tea polyphenol (-)-epigallocatechin gallate prevents the aggregation of tau protein into toxic oligomer at substoichiometric ratios. FEBS Lett. 589(1), 77-83 (2015).

82. Pickhardt $\mathrm{M}$, Gazova Z, von Bergen $\mathrm{M}$ et al. Anthraquinones inhibit tau aggregation and dissolve Alzheimer's paired helical filaments in vitro and in cells. J. Biol. Chem. 280(5), 3628-3635 (2005).

83. Caruana M, Högen T, Levib J et al. Inhibition and disaggregation of $\alpha$-synuclein oligomers by natural polyphenolic compounds. FEBS Lett. 585(8), 1113-1120 (2011).

84. Takahashi R, Ono K, Takamura Y et al. Phenolic compounds prevent the oligomerization of $\alpha$-synuclein and reduce synaptic toxicity. J. Neurochem. 134(5), 943-955 (2015).

85. Jha NN, Gosh D, Das S et al. Effect of curcumin analogs on $\alpha$-synuclein aggregation and cytotoxicity. Sci. Rep. 6, 28511 (2016).

86. Singh PK, Kotia V, Ghosh D et al. Curcumin modulates $\alpha$-synuclein aggregation and toxicity. ACS Chem. Neurosci. 4(3), 393-407 (2013).

- Describes how curcumin, the most promising phytochemical, suppressed the cytotoxicity of $\alpha$ Syn oligomer and the aggregation.

87. Cheung ZH, Ip NY. Autophagy deregulation in neurodegenerative disease - recent advances and future perspectives. $J$. Neurochem. 118(3), 317-325 (2011).

88. Menzies FM, Fleming A, Caricasole A et al. Autophagy and neurodegeneration: pathogenic mechanisms and therapeutic opportunities. Neuron 93(5), 1015-1034 (2017).

-. Presents the mechanism behind the association of autophagy with neurodegeneration and the therapeutic targets in autophagic process.

89. Guo YJ, Dong SY, Cui XX et al. Resveratrol alleviates MPTP-induced motor impairments and pathological changes by autophagic degradation of $\alpha$-synuclein via SIRT1-deacetlated LC3. Mol. Nutr. Food Res. 60(10), 2161-2175 (2016).

90. Jiang TF, Zhang YJ, Zhou HY et al. Curcumin ameliorates the neurodegenerative pathology in A53T $\alpha$-synuclein cell death of Parkinson's disease through the downregulation of mTOR/p70S6K signaling and the recovery of macroautophagy. J. Neuroimmune Pharmacol. 8(1), 356-369 (2013).

91. Wang C, Zhng X, Teng Z, Zhang T, Li Y. Downregulation of PI3K/Akt/mTOR signaling pathway in curcumin-induced autophagy in APP/PS1 double transgenic mice. Eur. J. Pharmacol. 740, 312-320 (2014).

92. Cordero JG, Garcia-Escudero R, Avila J, Gargini R, Garcia-Escudero V. Benefit of oleuropein aglycone for Alzheimer's disease by promoting autophagy. Oxid. Med. Cell. Longev. 2018, 5010741 (2018).

93. Wu Y, Li X, Zhu JX et al. Resveratrol-activated AMPK/SIRT1/autophagy in cellular models of Parkinson's disease. Neurosignals 19, 163-174 (2011).

94. Zhang L, Fang Y, Cheng X et al. The potential protective effects of curcumin on amyloid- $\beta$ - 42 induced cytotoxicity in HT-22 cells. BioMed. Res. Inter. 2018, 8134902 (2018).

95. Pandareesh MD, Mythri RB, Srinivas Bharath MM. Bioavailability of dietary polyphenols: factors contributing to their clinical application in CNS diseases. Neurochem. Int. 89, 198-208 (2015). 
96. Gomes NG, Campos MG, Orfäo JM, Ribeiro CA. Plants with neurobiological activity as potential targets for drug discover. Prog. Neuropsychopharmacol. Biol. Psychiatry 33(8), 1372-1389 (2009).

97. Pandareesh MD, Myrthri RB, Srinivas Bharath MM. Bioavailability of dietary polyphenols: factors contributing to their clinical application in CNS diseases. Neurochem. Int. 89, 198-208 (2015).

98. Engelbrecht I, Petzer JP, Petzer A. The synthesis and evaluation of sesamol and benzodioxane derivatives as inhibitors of monoamine oxidase. Bioorg. Med. Chem. Lett. 25(9), 1896-1900 (2015).

99. Badavath VN, Baysal I, Ucar G, Sinha BN, Jayaprakash V. Monoamine oxidase inhibitory activity of novel pyrazoline analogues: curcumin based design and synthesis. ACS Med. Chem. Lett. 7(1), 56-61 (2016).

100. Xie SS, Wang X, Jiang $\mathrm{N}$ et al. Multi-target tacrine-coumarin hybrids: cholinesterase and monoamine oxidase inhibition properties against Alzheimer's disease. Eur. J. Med. Chem. 95, 153-165 (2015).

101. Sun Y, Chen J, Chen X, Huang L, Li X. Inhibition of cholinesterase and monoamine oxidase-B activity by tacrine-homoisoflavonoid hybrid. Bioorg Med. Chem. 21(23), 74016-7417 (2013).

102. Li Y, Qiang X, Luo L et al. Multitarget drug design strategy against Alzheimer's disease: homoisoflavonoid Mannich base derivatives serve as acetylcholinesterase and monoamine oxidase B dual inhibitors with multifunctional properties. Bioorg. Med. Chem. 25(2), 714-726 (2017).

103. Amat-ur-Rasool H, Ahmed M. Designing second generation anti-Alzheimer compounds as inhibitors of human acetylcholinesterase: computational screening of synthetic molecules and phytochemicals. PLoS ONE 10(9), e0136509 (2015).

104. Naoi M, Maruyama W, Shamoto-Nagai M. Type A monoamine oxidase and serotonin are coordinately involved in depressive disorders: from neurotransmitter imbalance to impaired neurogenesis. J. Neural Transm. 125(1), 53-66 (2018).

105. Lopresti AL, Drummond PD. Efficiency of curcumin, and a saffron/curcumin combination for the treatment of major depression: a randomised, double-blind, placebo-controlled study. J. Affect. Disord. 207, 188-196 (2017).

106. Dalla Via L, Garcia-Argaez AN, Martinez-Vazquez M et al. Mitochondrial permeability transition as target of anticancer drugs. Curr. Pharm. Des. 20(2), 223-244 (2010). 
